\title{
Hole-Phonon Relaxation and Photocatalytic Properties of Titanium Dioxide and Zinc Oxide: First-Principles Approach
}

\author{
V. P. Zhukov, ${ }^{1,2}$ V. G. Tyuterev, ${ }^{3,4}$ E. V. Chulkov, ${ }^{2,5,6}$ and P. M. Echenique ${ }^{2,5}$ \\ ${ }^{1}$ Institute of Solid State Chemistry, Urals Branch of the Russian Academy of Sciences, Pervomayskaya 91, Yekaterinburg 620990, Russia \\ ${ }^{2}$ Donostia International Physics Center (DIPC), P. Manuel de Lardizabal 4, 20018 San Sebastián, Spain \\ ${ }^{3}$ Tomsk State Pedagogical University, Kievskaya 60, Tomsk 634041, Russia \\ ${ }^{4}$ National Research Tomsk State University, Lenin Street 36, Tomsk 634050, Russia \\ ${ }^{5}$ Departamento de Física de Materiales, Facultad de Ciencias Químicas, UPV/EHU and CFM-MPC, Apartado 1072, \\ 20080 San Sebastián, Spain \\ ${ }^{6}$ Tomsk State University, Lenina 36, Tomsk 634050, Russia
}

Correspondence should be addressed to V. P. Zhukov; zhukov_vladlen@mail.ru

Received 27 February 2014; Accepted 17 March 2014; Published 14 April 2014

Academic Editor: Jiaguo Yu

Copyright (C) 2014 V. P. Zhukov et al. This is an open access article distributed under the Creative Commons Attribution License, which permits unrestricted use, distribution, and reproduction in any medium, provided the original work is properly cited.

\begin{abstract}
First-principles calculations for the temporal characteristics of hole-phonon relaxation in the valence band of titanium dioxide and zinc oxide have been performed. A first-principles method for the calculations of the quasistationary distribution function of holes has been developed. The results show that the quasistationary distribution of the holes in $\mathrm{TiO}_{2}$ extends to an energy level approximately $1 \mathrm{eV}$ below the top of the valence band. This conclusion in turn helps to elucidate the origin of the spectral dependence of the photocatalytic activity of $\mathrm{TiO}_{2}$. Analysis of the analogous data for $\mathrm{ZnO}$ shows that in this material spectral dependence of photocatalytic activity in the oxidative reactions is unlikely.
\end{abstract}

\section{Introduction}

The relaxation of excited holes in the photocatalytic oxides is manifested in the transfer of holes to the highest valence band states and in trapping them on defects or surface states, the processes that profoundly affect the properties of the oxides [1-10]. Recently, Henderson published a review studies concerning the relaxation [11]; therefore, we restrict our introduction only to studies associated with the relaxation of holes that occurs via interactions with phonons and that terminates when holes ascend to the top of the valence band.

In photocatalytic oxides, the thermalization of excited electrons via electron-phonon interaction occurs very rapidly, within several hundreds of femtoseconds; see references in $[12,13]$. It is natural to suppose that the relaxation of excited holes in the valence band also occurs rapidly. Since the electron-hole recombination is a much slower process, one can assume that the efficiency of a photocatalyst should not depend on the energy of the exciting photon [11]. However, this assumption is not true.
The dependence of photocatalytic activity on the energy of excitation has been observed for the first time by Grela et al. [1] who studied the photocatalytic oxidation of salicylate (S) in presence of aqueous sols containing nanoparticles of $\mathrm{TiO}_{2}$. The authors found that the photons absorbed through nanoparticles at $254 \mathrm{~nm}$ were 8-times more efficient than those absorbed at $366 \mathrm{~nm}$. The authors proposed that the thermalization of holes in nanoclusters occurs in the nanosecond timescale compared with the timescale of hole transitions to the molecules absorbed on the surface. The authors subsequently studied the photocatalysis of salicylate, phthalate (P), and resorcinol (R) [3]. It is well known that the effective mass of excited electrons is greater than the mass of holes, so the authors suggested that the excess photon energy is channeled into holes. An essential finding of this work is that the quantum yield of the oxidative reaction suddenly increases when the excess energy increases above $\sim 0.2 \mathrm{eV}$. The maximum quantum yield is achieved at $0.5 \mathrm{eV}$ for $\mathrm{P}$, but the yield rises permanently for $\mathrm{S}$ and remains constant for $\mathrm{R}$. These trends are explained by the Marcus theory [14-16], and 
these results suggest that hole relaxation is a slower process compared with hole transfer from a solid to a molecular state; otherwise the variation of the quantum yield with the excess energy would be impossible.

Emeline et al. [5] studied the effects of photodegradation of phenol and 4-chlorophenol on the nanoparticles of the standard Degussa photocatalyst, confirming the spectral dependence of the photocatalytic activity. These authors observed that the quantum yield of the reactions varies with changes in the photon energy, as the variation of quantum yield has at least three maxima at energies corresponding to direct and indirect electron excitations in bulk $\mathrm{TiO}_{2}$. Authors related these changes to the mobility and lifetimes of the holes generated at different excitation energies in different points of the Brillouin zone. Notably, the photocatalyst nanoparticles obtained in the experiments of Emeline et al. $(\sim 30 \mathrm{~nm})$ were markedly larger than the nanoparticles obtained in the studies of Grela et al. ( $5 \mathrm{~nm})$. These circumstances imply that spectral dependence of the photocatalytic activity can be a property of both nanoparticles and bulk photocatalysts.

Although the authors of the cited papers emphasized the important role of the hole-phonon relaxation, they did not evaluate the temporal characteristics of these processes. To our knowledge, only Morishita et al. [4] have attempted such evaluations. In their work the two-photon femtosecond transient reflecting grating (TRG) method was applied to determine the dynamics of the currents for the degradation of KSCN on the (001) surface of $\mathrm{TiO}_{2}$. The authors showed that the dependence of the probe TRG-signal on the delay between the pump and probe pulses reflects changes in the concentration of holes excited by the pump. They fitted the signal to two exponential curves, a slow and a quick one. The slow component, with a time constant from 150 to 173 ps, has been attributed to electron-hole recombination. The quick component, whose intensity depends on the surface concentration of KSCN and intensity of irradiation, was attributed to the decrease in the hole concentration due to electron transfer from KSCN to the holes in $\mathrm{TiO}_{2}$. With increasing pump power intensity the time constant of the quick component varied from 110 to $690 \mathrm{fs}$. The time constant of the holephonon relaxations cannot be less than the time constant of the quick component; otherwise the quick component is absent. So these results help to estimate the time constant of the hole relaxation.

In the remaining papers cited at the onset $[6,8-10]$, the processes of carrier trapping on the defects or on the surface states have been discussed, although these processes are too slow to be compared with the time of the holephonon relaxation. Hence, despite the important role of the hole-phonon relaxation, the available data on the timing of these processes are scanty and insufficiently accurate. The number of theoretical works on this subject is limited and incomparable to the numerous studies of relaxation time in metals [17-19]. The formerly developed methods and computer codes for the solid state theory [20] facilitate firstprinciple calculations for the temporal characteristics of the relaxation processes. However, to our knowledge, only in the works $[12,13,21]$ the calculations for the relaxation time of excited electrons in $\mathrm{TiO}_{2}$ and $\mathrm{ZnO}$ have been performed.

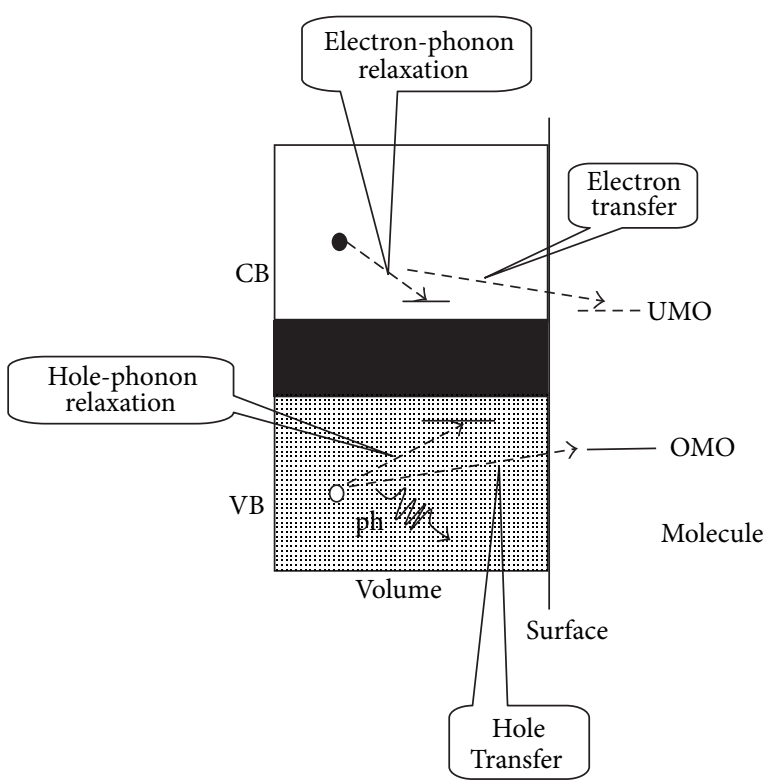

FIGURE 1: The scheme of the processes that occur after the excitation of the electron-hole pairs near the surface of a photocatalyzer. The notations have the following sense: VB is the band of valence states, $\mathrm{CB}$ is the band of conduction states, and OMO and UMO are the occupied and unoccupied molecular states, respectively.

The calculated times of electron-phonon relaxation in these studies are fairly consistent with the experimental data, and the distinctions are explained through the specificity of the performed experiments.

Therefore, we have carried out a first-principles study for the processes of relaxation of the nonequilibrated holes in $\mathrm{TiO}_{2}$ and $\mathrm{ZnO}$. In what follows we outline the physical foundations of the method and the details of the calculations and discuss the results and conclusions important for a better understanding of the photocatalytic properties of these oxides.

\section{Theory}

2.1. The Rate of Hole-Phonon Relaxation and the Time of Energy Loss. In Figure 1 we show the scheme of the processes that occur near the surface of a photo-catalyzer just after an electron-hole pair has been excited by the light. Both the excited hole in the valence band and the electron in the conduction band lose their energy by phonon emission. Simultaneously, the electron and hole transfer from the volume of the photo-catalyzer to the molecules adsorbed on the surface can take place. It has been shown in the work [2] that more than $98 \%$ of the photon energy goes into excitation of the holes, so we discuss in the following the rate of the hole-phonon scattering. One of our aims is to evaluate the time of the hole energy loss. The spectral dependence of the photocatalytic activity can be observed if only this time is longer than the time of hole transfer to the molecule. So the time of hole energy loss is the high limit of the hole transfer time. 
Our evaluation of the hole energy loss time is based on the "golden Fermi rule" of the perturbation theory. Consistent with this rule, the probability, per unit of time, of an elementary transition of a single electron from the state with the wave-vector $\mathbf{k}$ and energy $e_{\mathbf{k}, i}$ to the state with the wave-vector $\mathbf{k}+\mathbf{q}$ and energy $e_{\mathbf{k}+\mathbf{q}, j}$, without considering the thermodynamic factors, can be written as

$$
\begin{aligned}
P_{\mathbf{k}, i ; \mathbf{k}+\mathbf{q}, j}^{\mathbf{q} v}= & \left(\frac{2 \pi}{\hbar}\right)\left|\left\langle\mathbf{k}, i\left|\Delta V_{\mathbf{q} \nu}\right| \mathbf{k}+\mathbf{q}, j\right\rangle\right|^{2} \\
& \times \delta\left(e_{\mathbf{k}, i}-e_{\mathbf{k}+\mathbf{q}, j} \pm \hbar \omega_{\mathbf{q}}^{\nu}\right) .
\end{aligned}
$$

Here, + and - represent the transitions accompanied by the absorption or the emission, respectively, of a phonon, $|\mathbf{k}, i\rangle$ and $|\mathbf{k}+\mathbf{q}, j\rangle$ are the wave functions of the electronic states, $\mathbf{q}$ and $v$ are the wave vector and polarization of the phonon, and $\Delta V_{\mathbf{q} v}$ is the variation of the self-consistent potential in the crystal caused by the displacement mode of the phonon. The value $\left\langle\mathbf{k}, i\left|\Delta V_{\mathbf{q} v}\right| \mathbf{k}+\mathbf{q}, j\right\rangle$ is the matrix element of the electron-phonon interaction; the ways of calculating this matrix element have been widely discussed [20]. The energy of phonon is typically neglected, as this value is small compared with the energies of electronic states $e_{\mathbf{k}+\mathbf{q}, j}$ and $e_{\mathbf{k}, j}$, representing the so-called quasielastic approximation. After integrating over all wave vectors and polarizations of the phonons and energies of electronic states, the probability is obtained, satisfying the momentum and energy conservation, for the electron to leave the state $|\mathbf{k}, i\rangle$, losing the energy $\hbar \omega$ :

$$
\begin{aligned}
\Phi_{\mathbf{k}, i}(\omega)=\left(\frac{2 \pi}{\hbar}\right) \sum_{j} \sum_{\nu} \int_{B Z} & \delta\left(\omega_{\mathbf{q}}^{v}-\omega\right)\left|\left\langle\mathbf{k}, i\left|\Delta V_{\mathbf{q} v}\right| \mathbf{k}+\mathbf{q}, j\right\rangle\right|^{2} \\
& \times \delta\left(e_{\mathbf{k}+\mathbf{q}, j}-e_{\mathbf{k}, i}\right) d^{3} \mathbf{q} .
\end{aligned}
$$

Function $\Phi_{\mathbf{k}, i}(\omega)$ is an analog of the well-known Eliashberg function $\alpha^{2} F_{\mathbf{k}, i}(\omega)$ [22]. The difference is in a constant,

$$
\alpha^{2} F_{\mathbf{k}, i}(\omega)=\frac{\Phi_{\mathbf{k}, i}(\omega)}{(2 \pi / \hbar)}
$$

In addition, the conventional Eliashberg function is defined at the Fermi energy of metals, whereas $\Phi_{\mathbf{k}, i}(\omega)$ is defined at arbitrary energy inside the valence band. The Eliashberg function is connected with a constant of electron-phonon coupling $\lambda$, calculated as

$$
\lambda_{\mathbf{k}, i}=2 \int \frac{\alpha^{2} F_{\mathbf{k}, i}(\omega)}{\omega} d \omega .
$$

When the thermodynamic equilibrium exists, the change in the population of the state at energy $e_{\mathbf{k}, i}$ is determined by the effects of the four kinds of electron transitions that occur between the $e_{\mathbf{k}, i}$-state and the states at $e_{\mathbf{k}, i} \pm \hbar \omega$ and are accompanied by the phonon absorption or emission. The probability of these processes, hereafter $U$, is determined by the probability of the elementary process and Bose statistics $Q(\hbar \omega, T)$ :

$$
\begin{aligned}
U_{\mathbf{k}, i ; \mathbf{k}+\mathbf{q}, j}^{\mathbf{q} v}= & P_{\mathbf{k}, i ; \mathbf{k}+\mathbf{q}, j}^{\mathbf{q} v}\left[Q\left(\hbar \omega_{\mathbf{q} v}, T\right)+I\right] \\
& \times \delta\left(e_{\mathbf{k}, i}-e_{\mathbf{k}+\mathbf{q}, j} \pm \hbar \omega_{\mathbf{q} v}\right)
\end{aligned}
$$

where $I=0$ represents the absorption of phonon and 1 represents the emission. Considering this statistic for phonons and Fermi statistics for electrons, hereafter $R$, and assuming, for the dynamics of holes, that the initial population of the level is zero, an expression for the change of population rate (see details in [23]) can be obtained:

$$
\begin{gathered}
\Gamma_{\mathbf{k}, i}=\int d \omega \Phi_{\mathbf{k}, i}(\omega)\left[1+2 Q(\hbar \omega, T)+R\left(e_{\mathbf{k}, i}+\hbar \omega, T\right)\right. \\
\left.-R\left(e_{\mathbf{k}, i}-\hbar \omega, T\right)\right] .
\end{gathered}
$$

If the electron population is $n\left(e_{\mathbf{k}, i}\right)$, then the number of holes in the state is $n_{h}\left(e_{\mathbf{k}, i}\right)=1-n\left(e_{\mathbf{k}, i}\right)$, and so expression (6) also determines the rate of the hole relaxations. At low temperatures, this value is reduced with high precision to

$$
\begin{aligned}
\Gamma_{\mathbf{k}, i}=\int d \omega \Phi_{\mathbf{k}, i}(\omega)[2 Q & (\hbar \omega, T)+1]=\left(\frac{2 \pi}{\hbar}\right) \\
\times \sum_{j} \sum_{\nu} \int d \omega \int_{B Z} & d^{3} \mathbf{q} \delta\left(\omega_{\mathbf{q} \nu}-\omega\right) \\
& \times\left|\left\langle\mathbf{k}, i\left|\Delta V_{\mathbf{q} \nu}\right| \mathbf{k}+\mathbf{q}, j\right\rangle\right|^{2} \\
& \times \delta\left(e_{\mathbf{k}+\mathbf{q}, j}-e_{\mathbf{k}, i}\right)[2 Q(\hbar \omega, T)+1] .
\end{aligned}
$$

In the real calculations described below we neglect the $Q$ factor; hence we perform evaluations for the case of low temperatures.

Having calculated the rate of hole-phonon relaxation, we can evaluate the relaxation time of the hole defined as the time from the moment of its emergence to the moment of transition to a higher level, owing to the filling of the holecontaining state with an electron that emits phonon:

$$
\tau_{\mathbf{k}, i}=\frac{\hbar}{\Gamma_{\mathbf{k}, i}} .
$$

Based on the calculations of the relaxation rate, one can evaluate for the state $|\mathbf{k}, i\rangle$ the energy loss per unit of time, defined by the first moment of the probability function $\Phi_{\mathbf{k}, i}$ :

$$
\begin{aligned}
\Delta \widetilde{e}_{\mathbf{k}, i} & =\int d \omega \Phi_{\mathbf{k}, i}(\omega) \hbar \omega[2 Q(\hbar \omega, T)+1] \\
& =2 \pi \int d \omega \alpha^{2} F_{\mathbf{k}, i}(\omega) \hbar \omega[2 Q(\hbar \omega, T)+1]
\end{aligned}
$$




$$
\begin{aligned}
&=2 \pi \int d \omega \hbar \omega \\
& \times \sum_{j} \sum_{\nu} \int_{B Z} d^{3} \mathbf{q} \delta\left(\omega_{\mathbf{q}}^{\nu}-\omega\right)\left|\left\langle\mathbf{k}, i\left|\Delta V_{\mathbf{q} v}\right| \mathbf{k}+\mathbf{q}, j\right\rangle\right|^{2} \\
& \quad \times \delta\left(e_{\mathbf{k}+\mathbf{q}, j}-e_{\mathbf{k}, i}\right) \times\left[2 Q\left(\hbar \omega_{\mathbf{q} v}, T\right)+1\right] .
\end{aligned}
$$

We calculated the characteristics averaged over the wave vectors as

$$
\begin{aligned}
\lambda(E) & =\left[\frac{1}{N}(E)\right] \sum_{i} \int d^{3} \mathbf{k} \delta\left(e_{\mathbf{k}, i}-E\right) \lambda_{\mathbf{k}, i} \\
\Gamma(E) & =\left[\frac{1}{N}(E)\right] \sum_{i} \int d^{3} \mathbf{k} \delta\left(e_{\mathbf{k}, i}-E\right) \Gamma_{\mathbf{k}, i} \\
\Delta \widetilde{e}(E) & =\left[\frac{1}{N}(E)\right] \sum_{i} \int d^{3} \mathbf{k} \delta\left(e_{\mathbf{k}, i}-E\right) \Delta \widetilde{e}_{\mathbf{k}, i},
\end{aligned}
$$

where $N(E)$ are the densities of electronic states. Employing the calculated $\Gamma_{\mathbf{k}, i}$ and $\Delta \widetilde{e}_{\mathbf{k}, i}$, we can also evaluate the mean energy of the emitted phonons:

$$
\Delta e(E)=\frac{\sum_{i} \int d^{3} \mathbf{k} \delta\left(e_{\mathbf{k}, i}-E\right) \Delta \widetilde{e}_{\mathbf{k}, i}}{\sum_{i} \int d^{3} \mathbf{k} \delta\left(e_{\mathbf{k}, i}-E\right) \Gamma_{\mathbf{k}, i}}
$$

In the theory of photocatalytic processes, the energy loss time $\tau_{\text {en }}\left(E^{\prime}, E\right)$ can also be useful that we define as the average time necessary for a hole to pass from its initial energy level $E$ to the level $E^{\prime}$. If at any intermediate energy $E^{\prime \prime}$, the average relaxation time is $\tau\left(E^{\prime \prime}\right)$ and the average energy loss is $\Delta e\left(E^{\prime \prime}\right)$, then the rate of the energy loss at this energy level is $\Delta e\left(E^{\prime \prime}\right) / \tau\left(E^{\prime \prime}\right)=\Delta e\left(E^{\prime \prime}\right) \Gamma\left(E^{\prime \prime}\right)$. Then the energy loss time is

$$
\tau_{\mathrm{en}}\left(E^{\prime}, E\right)=\int_{E^{\prime}}^{E} \frac{d E^{\prime \prime}}{\Delta e\left(E^{\prime \prime}\right) \Gamma\left(E^{\prime \prime}\right)} .
$$

\subsection{Quasistationary Distribution of Holes inside the Valence} Band. Initially, we consider the temporal evolution of the mean population $n(t, E)$ of a single state at the energy level $E$. Considering the four aforementioned processes, this population can be written as (here $\epsilon \equiv \hbar \omega$ )

$$
\begin{aligned}
& \frac{\partial n(t, E)}{\partial t} \\
& =[1-n(t, E)] \int_{0}^{\hbar \omega_{m}} d \epsilon n(t, E+\epsilon) F(E+\epsilon, E)[Q(\epsilon)+1] \\
& \quad+[1-n(t, E)] \int_{0}^{\hbar \omega_{m}} d \epsilon n(t, E-\epsilon) F(E-\epsilon, E) Q(\epsilon) \\
& \quad-n(t, E) \int_{0}^{\hbar \omega_{m}} d \epsilon[1-n(t, E+\epsilon)] F(E, E+\epsilon) Q(\epsilon)
\end{aligned}
$$

$$
\begin{aligned}
& -n(t, E) \int_{0}^{\hbar \omega_{m}} d \epsilon[1-n(t, E-\epsilon)] F(E, E-\epsilon)[Q(\epsilon)+1] \\
& +\frac{\partial n^{\mathrm{ext}}(t, E)}{\partial t}
\end{aligned}
$$

In this equation the term $\partial n^{\text {ext }}(t, E) / \partial t$ describes the instantaneous hole distribution in the valence band produced by the source of light; its form is specified below. Function $F\left(E, E^{\prime}\right)$ (not to mix with the Eliashberg function!) is the probability of the elementary process of electron transition from the energy level $E$ to the level $E^{\prime}$ accompanied by the emission or absorption of the quantum $\epsilon=\left|E^{\prime}-E\right|$ summarized over all the states at these levels. It can be represented as

$$
\begin{aligned}
F\left(E, E^{\prime}\right)=\sum_{\mathbf{q} \mathbf{k k}^{\prime} n \sum^{\prime} \sigma} & \delta\left(E-e_{\mathbf{k}, n}\right) P_{\mathbf{k}, n ; \mathbf{k}^{\prime}, n^{\prime}}^{\mathbf{q} \sigma} \delta_{\mathbf{k}-k^{\prime}+\mathbf{q}} \\
& \times \delta\left(E^{\prime}-E \mp \epsilon_{\mathbf{q} \sigma}\right) \delta\left(E^{\prime}-e_{\mathbf{k}^{\prime}, n^{\prime}}\right) .
\end{aligned}
$$

We neglect the probability of the electron-hole recombination, as this process is to some extent slower. Besides, we apply the effective phonon approximation to these equations; that is, we approve that the phonon emission at all the same frequency with the same probability takes place; then

$$
F(E, E \pm \epsilon)=N(E) N(E \pm \epsilon) P(E) \delta\left(\epsilon-\hbar \omega_{0}\right),
$$

where $\hbar \omega_{0}$ is the energy of effective phonon. For the value we can use the average energy of the emitted phonon: $\hbar \omega_{0}(E)=$ $\Delta e(E)$. Studies $[12,13]$ have shown based on first-principle evaluations that in the cases of excited electrons in anatase, rutile, and zinc oxide the energy dependence of this value is unimportant, and this energy is close to the energy of the optic phonons. We will show below that for holes in the valence band the energy dependence is also insignificant.

Looking for an approximation for averaged transition probability $P(E)$ we use (1). This equation includes summation over electronic states only in narrow interval near the energy $e_{\mathbf{k}, n}$. Neglecting this energy interval, we assume that the mean transition probability can be approximated by

$$
P(E)=\frac{\Gamma(E)}{N(E)} .
$$

We define the total distribution function of electrons as the population of all the states at an energy level; hence it is $f(t, E)=N(E) n(t, E)$. When we neglect the terms proportional to $Q$, that is, when we consider the case of low temperatures, and linearize (15) with respect to the small parameter $\epsilon$, then we derive the equation for $f(t, E)$

$$
\frac{\partial f(t, E)}{\partial t}=\frac{\partial}{\partial E}\left[N^{-1}(E) f(t, E) B(E)\right]+\frac{\partial f^{\mathrm{ext}}(t, E)}{\partial t} .
$$

Here the function

$$
B(E)=\int_{0}^{\omega_{m}} \hbar \omega F(E, E+\hbar \omega) d \omega
$$


describes the energy loss of electrons on the level $E$ through the phonon emission, connected with the $\Delta e$-value by the relation $\Delta e(E)=B(E) / N^{2}(E)$. Because the hole distribution function, defined in a similar way, is $f_{h}(t, E)=N(E)-f(t, E)$, then $(20)$ is also applicable to the calculations of the hole distribution. However, the term $\partial f_{h}^{\text {ext }}(t, E) / \partial t$ analogous to the last term of (15) should also be defined; we accept for this term the approximation

$$
\frac{\partial f_{h}^{\text {ext }}(t, E)}{\partial t}=S_{0}(t) S_{h}(E) .
$$

Here the factor $S_{0}(t)$, which is time-dependent, describes the concentration of the holes excited by light, and the function $S_{h}(E)$, hereafter named as instantaneous hole distribution function (IDF), describes the probability of hole excitations depending on the excess energy of hole $E$. Naturally, it should be normalized to unity: $\int_{0}^{E_{\text {exc }}} S_{h}(E) d E=1$ where $E_{\text {exc }}$ is the photon energy. Admitting that the rate of change of the $S_{0}(t)$-function is small, that is, excluding the case of pulse irradiation and assuming that $\partial f_{h}(t, E) / \partial t=0$ we come to the quasistationary solution for $(20)$ :

$$
f_{h}(t, E)=S_{0}(t) \frac{1}{\Delta e(E) N(E)} \int_{E}^{E_{m}} S_{h}\left(E^{\prime}\right) d E^{\prime},
$$

where $E_{m}$ is the maximum excess energy of the excited holes.

The IDF-function can be obtained from the electronic band structure calculations. In order to calculate this, one should summarize the probabilities of all possible direct excitations from the states at the $E$ level to the states at the $E+E_{\text {exc }}$ level. Thus, the unnormalized IDF can be written as

$$
S_{h}(E)=\sum_{\mathbf{k} n n^{\prime}} \delta\left(E-e_{n \mathbf{k}}\right) T\left(n \mathbf{k}, n^{\prime} \mathbf{k}\right) \delta\left(e_{n \mathbf{k}}+E_{\mathrm{exc}}-e_{n^{\prime} \mathbf{k}}\right),
$$

where $T\left(n \mathbf{k}, n^{\prime} \mathbf{k}\right)$ is the probability of transitions between the $|n \mathbf{k}\rangle$ and $\left|n^{\prime} \mathbf{k}\right\rangle$ states. In calculations we replace the $\delta$ functions with the normalized Gaussian functions whose width at the half-maximum is $0.01 \mathrm{eV}$.

In order to calculate the probabilities of excitations, we apply the atomic sphere approximation [24]. The essence of this approximation resides in replacing the integration over whole space with the integrations over atomic spheres whose total volume covers the total space of crystal. To describe the interaction between the crystal and the light, we apply to every atomic sphere the so-called dipole approximation [25]. With such an approximation, the Hamiltonian of interaction of an atom $S$ with electric field of the light $\mathbf{E}(\omega)$ has the form

$$
H_{S}=e \mathbf{D}_{S} \mathbf{E}(\omega),
$$

where $e \mathbf{D}_{S}=e \sum_{t} \mathbf{r}_{t}^{S}$ is the operator of the dipole moment of atom, and $\mathbf{r}_{t}^{S}$ is the radius of an electron with respect to the center of atomic sphere. That is, the interaction of electrons with the field is

$$
H(\omega)=e \sum_{t, S} \mathbf{r}_{t}^{S} \epsilon_{\mathbf{E}} E(\omega)
$$

We consider the case of a polycrystal, so after averaging over the angles we have

$$
T\left(n \mathbf{k}, n^{\prime} \mathbf{k}\right)=\frac{2 \pi}{\hbar} \cdot e^{2} \cdot(E(\omega))^{2} \frac{1}{3}\left|\sum_{t, S}\left\langle\mathbf{k}, i\left|\mathbf{r}_{t}^{S}\right| \mathbf{k}, j\right\rangle\right|^{2} .
$$

The details of this derivation have been previously described [25]. Formerly we employed such an approach in the studies of radiative trapping of excited electrons on B-, C-, and Nimpurities in anatase [26].

The numerical evaluations for the electron-phonon coupling have been done using the density-functional perturbation theory [20] implemented in the pseudopotential Quantum Espresso (QE) computer code [27], version 4.0.4. We have inserted in the code all the modifications necessary for our purposes. A plane-wave basis set with the energy cutoff of $50 \mathrm{Ry}$ was employed which is sufficient for the successful calculations of the phonon frequencies in rutile [28]. Before the calculations of the phonons and electronphonon interactions, the crystal structures were optimized. The perfectness of the crystal structure optimization is characterized by the final variations of the total force and total energy of the compounds. In our case these variations were no more than 0.0001 Ry/at.un. and 0.00003 Ry for the force and energy, respectively. The precision of the phonon calculations is affected also by the choice of the atomic pseudopotentials. Normally, in the calculations for the compounds containing $3 \mathrm{~d}$-atoms the semicore $3 \mathrm{~s}$ - and $3 p$-states are treated as valence ones. With such basis sets, the calculations of the momentum-averaged characteristics of electron-phonon coupling are extremely time-consuming. An effective way to accelerate the calculations is to eliminate the semicore bands. So we have generated for titanium the ultrasoft Rabe-Rappe-Kaxiras-Joannopoulos pseudopotential with Purdew-Burke-Ernzerhof exchange and correlation (RRKJ-PBE) and only with the 3d-and 4s-states in basis set. The standard RRKJ-PBE pseudopotential was employed also for oxygen atoms. The details of the calculations with such pseudopotentials have been described in our previous paper on the excited electron relaxation in rutile and anatase [12]. They demonstrate that our modifications lead only to the changes in the calculated phonon spectra and electronphonon coupling constants insignificant in the context of our discussions.

The electron excitation probabilities $T\left(n \mathbf{k}, n^{\prime} \mathbf{k}\right)$ were calculated based on the linear method of muffin-tin orbitals for the band structure calculations (LMTO-TB) computer code [29]. A well-known underestimation of the band gap inherent in this approach has been amended through the application of a single-site coulomb and exchange correction terms [30]. In order to calculate matrix elements of dipole moment the integration, procedures implemented in the GW computer code [31] have been employed.

\section{Results and Discussions}

In Figures 2, 3, and 4 we show the calculated values of $\lambda$, $\Gamma$, and $\Delta e$ as functions of the excess energy of the hole $E$ 


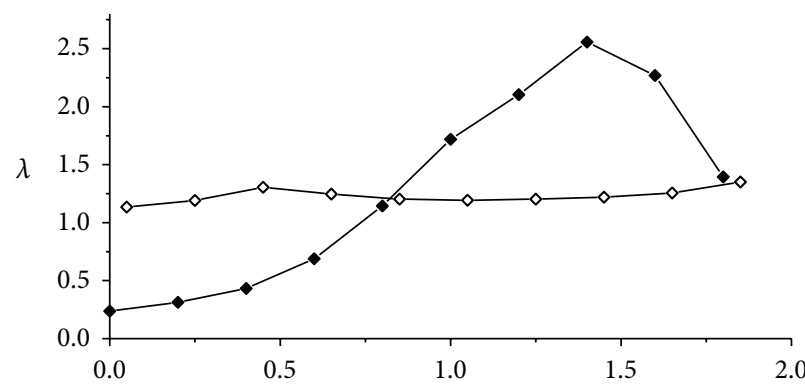

(a)

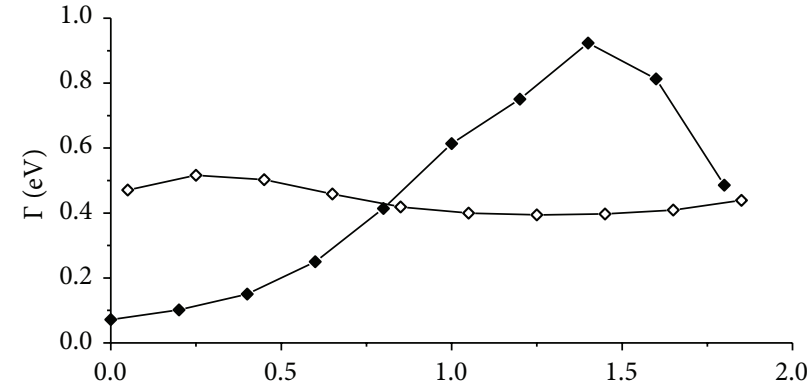

(b)

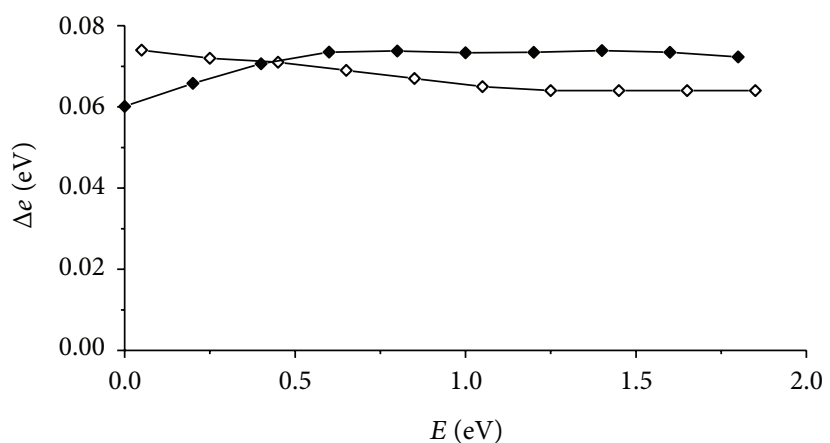

(c)

FIGURE 2: The mean characteristics of the hole-phonon (empty rhombus) and electron-phonon (black rhombus) relaxation for anatase as functions of the excess energy $E$. Here $\lambda$ is the constant of interaction, $\Gamma$ is the rate of energy loss according to (7) and (11), and $\Delta e$ is the mean energy lost with emission of one phonon, in accordance with (9), (12), and (13).

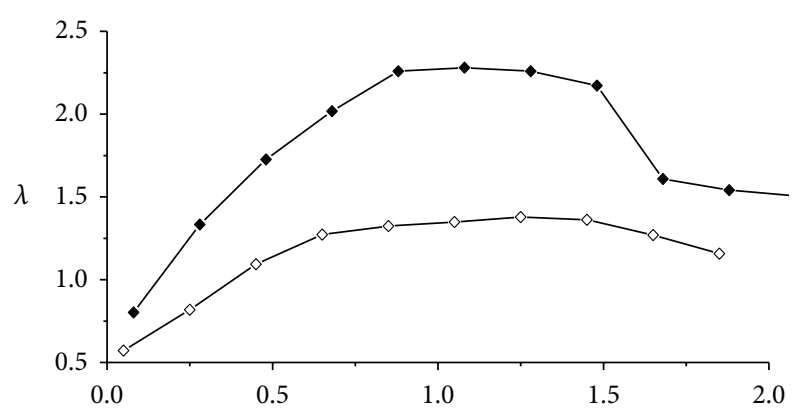

(a)

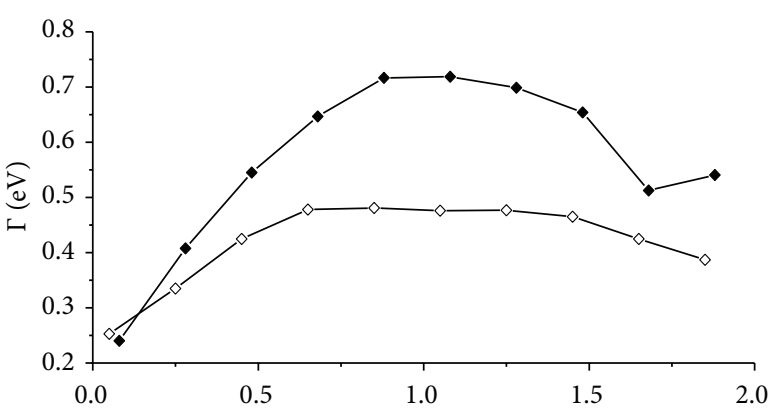

(b)

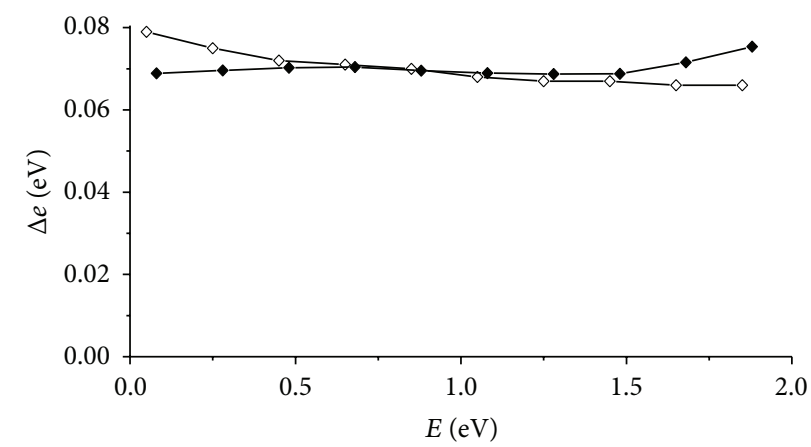

(c)

Figure 3: The characteristics of the hole-phonon and electron-phonon relaxation for rutile depending on the excess energy $E$. The sense of the plots is the same as in Figure 2. 


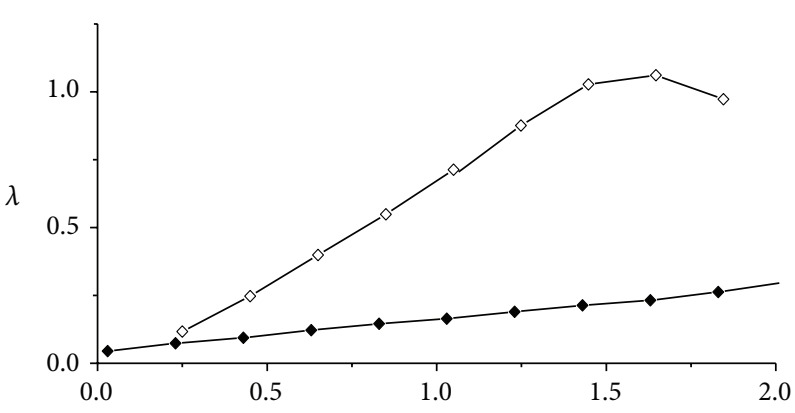

(a)

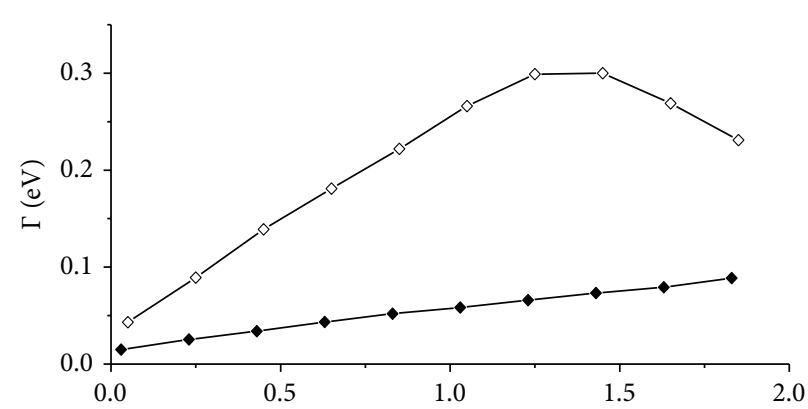

(b)

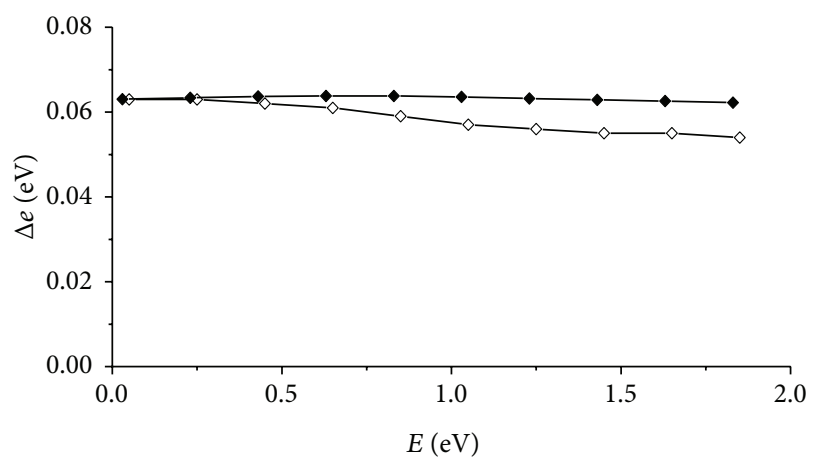

(c)

Figure 4: The characteristics of the hole-phonon and electron-phonon relaxation for ZnO depending on the excess energy E. The sense of the plots is the same as in Figure 2.

and compare them with the analogous values for excited electrons. For rutile, our results confirm the previously proposed assumption $[1,11]$ that the hole-phonon relaxation is a process slower than the electron-phonon relaxation. For anatase, however, the hole relaxation is slower than the electron relaxation only when the excess energy is higher than $0.7 \mathrm{eV}$. For $\mathrm{ZnO}$ the situation is strikingly different, as the rate of the hole relaxation is higher. In all the three cases the mean energy of the emitted phonon $\Delta e$ is close to the analogous value for electrons, and this value changes insignificantly with changes in the excess energy. Therefore, we admit in the calculations for the energy loss time and distribution function that this value is constant and equal to $70 \mathrm{meV}$ for anatase and rutile and $55 \mathrm{meV}$ for $\mathrm{ZnO}$.

In Figure 5 we show the time of energy loss of a hole, depending on its final energy $E^{\prime}$, as calculated using (14). This value has been calculated for the initial excess energy of the hole $E=2 \mathrm{eV}$ that was the highest energy in the experiments [1]. For holes in rutile, the energy loss time $\tau_{\text {en }}\left(E^{\prime}, E\right)$ is at any excess energy $\sim 30 \%$ longer than that for electrons. For holes in anatase the energy loss time is higher only when the excess energy is more than $0.5 \mathrm{eV}$. For holes in $\mathrm{ZnO}$ the energy loss time at any excess energy is about 3 times less than the electron energy loss time. So our results do not support the assumption that in the case of $\mathrm{TiO}_{2}$ the hole relaxation time is much longer than the electron relaxation time, and that was proposed in earlier papers in order to explain the existence of the spectral dependence of photocatalytic activity.
Results of our calculations for the distribution functions of holes are shown in Figure 6. For anatase, the concentration of holes near zero excess energy is accompanied with a tail that extends to the energy of approximately $1 \mathrm{eV}$, the concentration of holes takes place both near the top of the valence band and in the lower states as well. This effect is even more pronounced in rutile which exhibits a sharp rise in the distribution at the energy of $0.4-0.5 \mathrm{eV}$. In the case of $\mathrm{ZnO}$ the rise of the distribution function appears only below $0.2 \mathrm{eV}$, and the hole concentration in the states below the top of the valence band is rather weak. In particular, the hole distribution differs in a crucial way from the analogous distribution for electrons, inasmuch as the calculations demonstrate that the concentration of excited electrons takes place only near the edge of the conduction band [21].

It has been shown $[1,3]$ that the quantum yield of the processes of photodegradation of a series of aromatic compounds on the surface of $\mathrm{TiO}_{2}$ nanoparticles depends on the energy of the photon. In particular, with the rise of the excess energy an increase of the quantum yield is observed beginning from $E \sim 0.2 \mathrm{eV}$. At higher energy, depending on the type of molecule, this rise converts to a plateau or a maximum. These results definitely demonstrate that the holes with excess energy higher than $0.2-0.5 \mathrm{eV}$ participate in the oxidative reactions. This fact squares with the presence in our calculations of the distribution function that extend to $\sim 1 \mathrm{eV}$.

Because the specimens studied in experiments were mixtures of the anatase and rutile phases, the question did not arise concerning which phase was responsible for the 


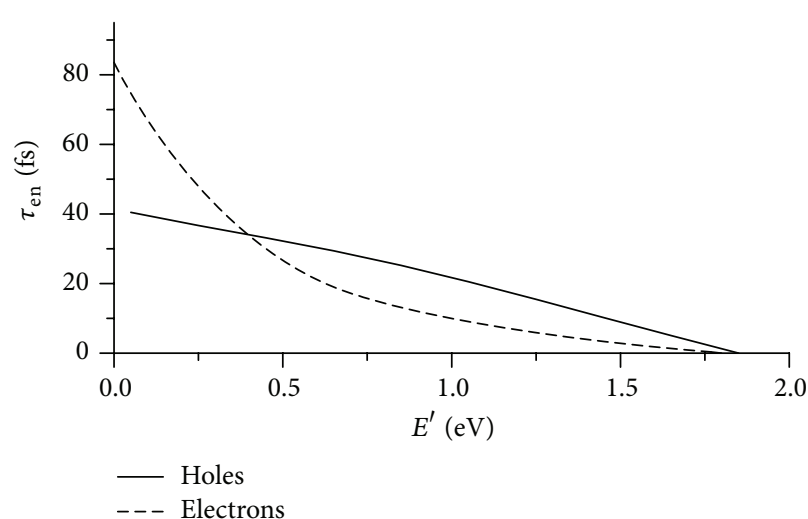

(a)

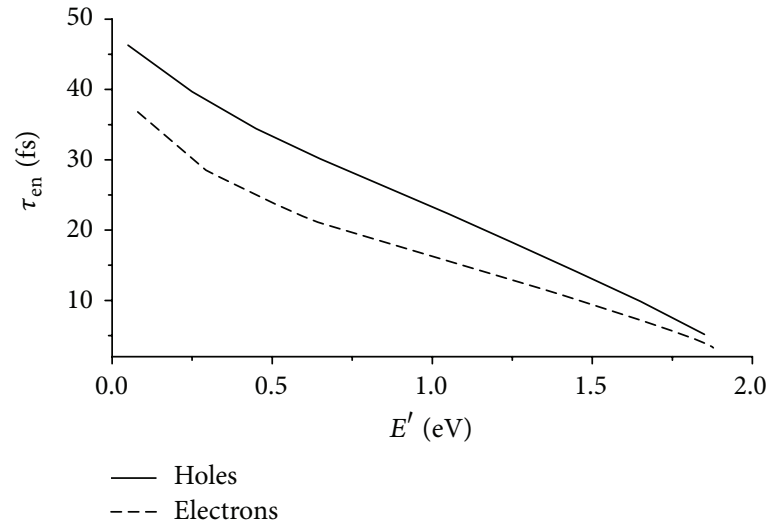

(b)

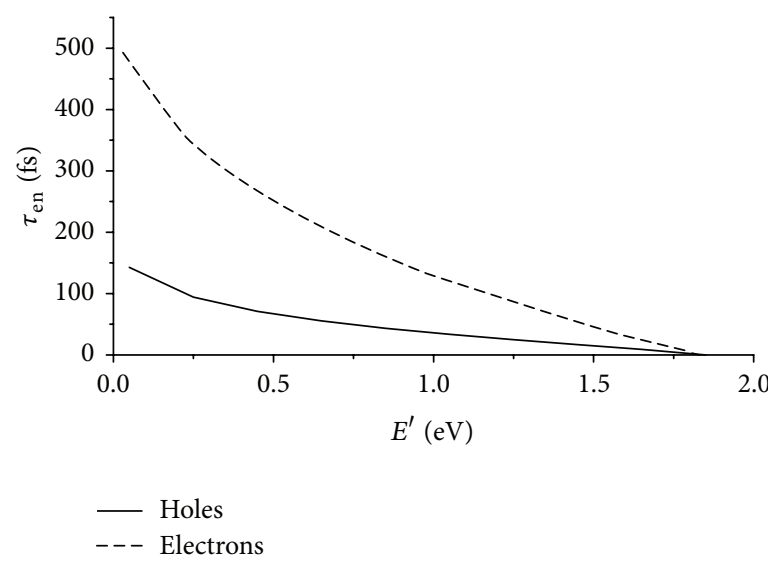

(c)

Figure 5: The time of energy loss of a hole (solid lines) depending on the final value of the excess energy $E^{\prime}$ for anatase (a), rutile (b), and zinc oxide (c). It is assumed that in the initial moment the hole was excited to the level $E=2 \mathrm{eV}$. For comparison, the analogous data are shown (dashed lines) for the energy loss time of excited electrons as calculated based on the results of the works [12, 13].

spectral dependence of the quantum yield. We can now turn our attention to the difference in the distribution functions of holes in the energy interval $0.2-0.7 \mathrm{eV}$. Here the value of this function is for rutile substantially higher than that for anatase. This difference indicates that rutile is the phase mostly responsible for the spectral dependence. To explain the differences in the distribution functions of anatase and rutile, let us examine the total densities of the states near the top of the valence band; see Figure 7. It is evident that, within the interval from 0 to $0.5 \mathrm{eV}$, the density of the states in anatase is much higher than that in rutile, consistently with (21), which generates smaller values for the distribution function. Physically, the higher distribution function of rutile reflects a much worse number of electrons that can drop to a hole state thus indicating its relaxation.

Our data indicate that zinc oxide is least favorable for the manifestation of spectral dependence of photocatalysis. The distribution function of holes in $\mathrm{ZnO}$ has a peak only below $0.2 \mathrm{eV}$, with no tail at a higher excess energy. The origin of the difference in the distribution functions of $\mathrm{TiO}_{2}$ and $\mathrm{ZnO}$ is concealed in the details of the IDF-function $S_{h}(E),(21)$. In Figure 8 we show the IDF calculated for photon energies from
3.4 to $4.6 \mathrm{eV}$. These results demonstrate that in the case of anatase and rutile the holes are produced in both the lower states and the states near the top of the valence band. In both cases, with increase in photon energy, the maxima of the IDF shift to higher excess energy. In the case of anatase, the main feature of the distribution is a high peak located near the excess energy $E$ that changes with the photon energy $E_{\text {exc }}$ approximately as $E=-2.0+0.66 E_{\text {exc }}$. This change is accompanied by spikes at lower $E$ that are much lower in height. In the case of rutile, the analogous dependence is more complicated. At excitation energies above $3.5 \mathrm{eV}$, up to seven peaks of comparable height, are observed. Thus, in this case, the holes are produced with comparable probability in many states below the top of the valence band.

In contrast, in the case of $\mathrm{ZnO}$, the holes are produced only in states near the top of the valence band. The IDF dependence has only one peak, which does not appear beyond $0.2 \mathrm{eV}$. In accordance with (22), the quasistationary distribution function also does not appear at the energy beyond this limit. Given this, the absence of excitations from the low-energy valence band states and spectral dependence of the oxidative reactions are unlikely in $\mathrm{ZnO}$. 


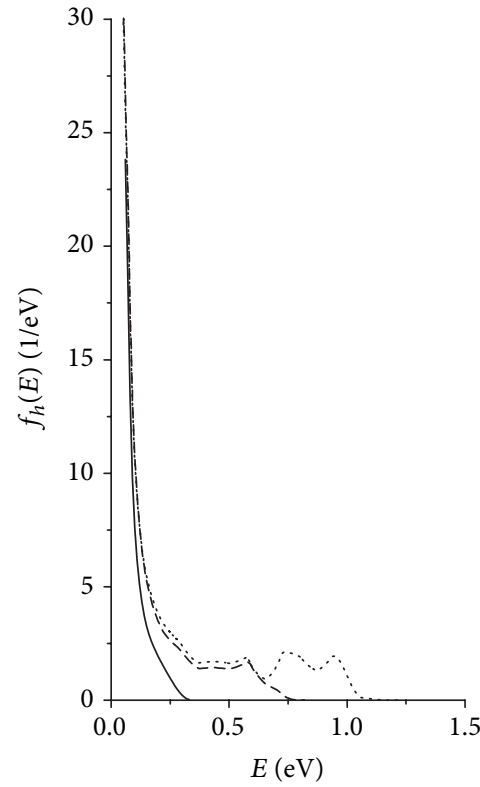

(a)

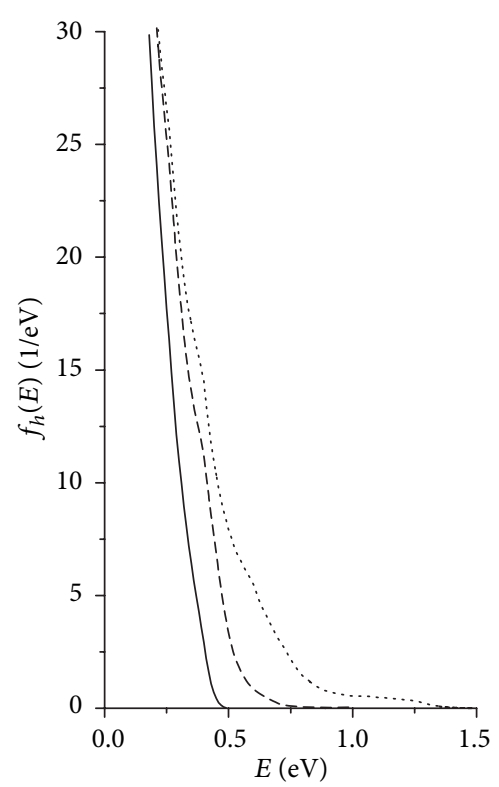

(b)

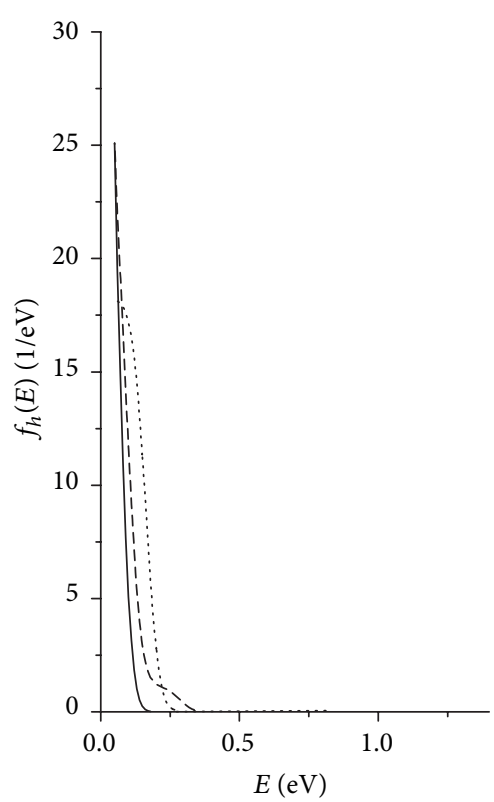

(c)

Figure 6: The calculated distribution function of holes in anatase (a), rutile (b), and zinc oxide (c). In solid lines the data are shown for the photon energy of $3.5 \mathrm{eV}$, in dashed lines the data are shown for $4.0 \mathrm{eV}$, and in point lines the data are shown for $4.5 \mathrm{eV}$. It is assumed that $S_{0}(t)=1$.

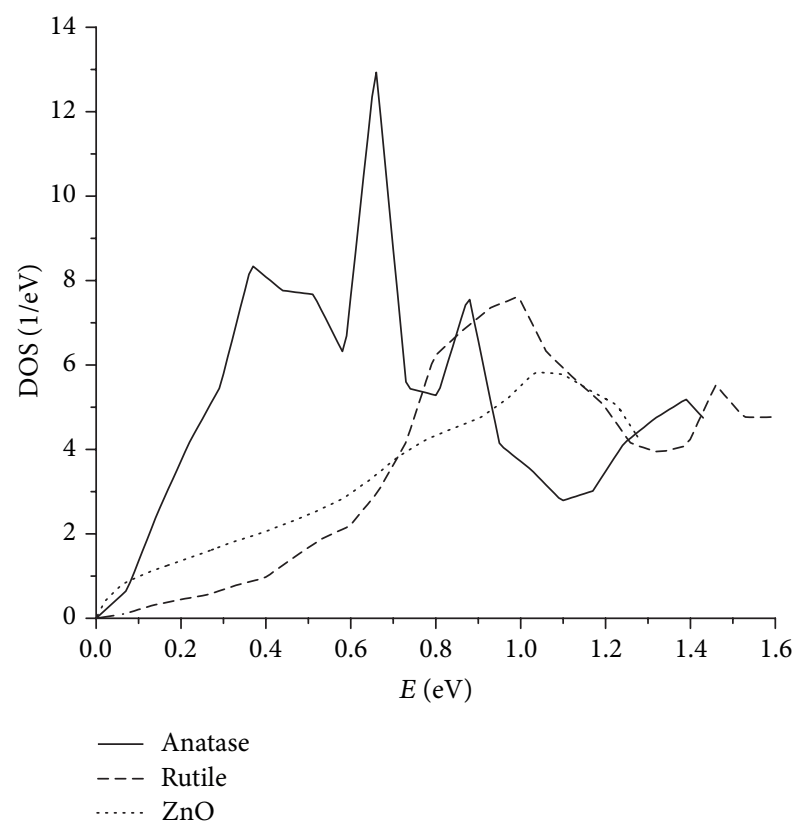

Figure 7: The total density of states near the top of the valence band versus the excess energy. The solid line is for anatase, the dashed line is for rutile, and the dotted line is for zinc oxide.

The results of our calculations make it possible to estimate the timescale of hole transfer from the bulk of the crystals to absorbed molecules. The experimental data $[2,3]$ demonstrate an increase in the rate of oxidative reactions when the excess energy of holes exceeds $0.2-0.5 \mathrm{eV}$. Our calculations for the hole distribution functions for $\mathrm{TiO}_{2}$ show that the holes can emerge in many states lower than the top of the valence band. If the time of the hole transfer to molecules is less than the time of the energy loss via coupling with phonons, the holes in the states below the top of the valence band cannot participate in oxidative reactions. Hence one can expect that the time of hole transfer should be less than $\sim 50 \mathrm{fs}$.

In order to compare this result with the experimental data of Morishita et al. [4], we need to take into account the essential differences between our first-principle and Morishita's experimental approach. We evaluate the relaxation rate of a single hole, but the results of Morishita et al. definitely demonstrate the dependence of the hole transfer time on the pump power intensity, that is, on the number of excited holes. Hence one should compare our estimation with the extrapolation of the Morishita's results to zero pump power. Because of the shortage of experimental data for low pump power, this extrapolation cannot be reliably performed. It is clear, however, that the extrapolated data should be lower than $100 \mathrm{fs}$.

Unfortunately, the experimental data on the hole relaxation rate in $\mathrm{ZnO}$ are absent. Also the absence of experimental data on the spectral dependence of the photocatalytic yields makes the evaluation of the hole transfer time in $\mathrm{ZnO}$ impossible now.

Note that first-principles calculations for the hole transfer time have not been performed.

\section{Conclusions}

We have performed first-principles calculations for characteristics of electron-phonon relaxation of excited holes in the valence band of $\mathrm{TiO}_{2}$ and $\mathrm{ZnO}$. These values are 


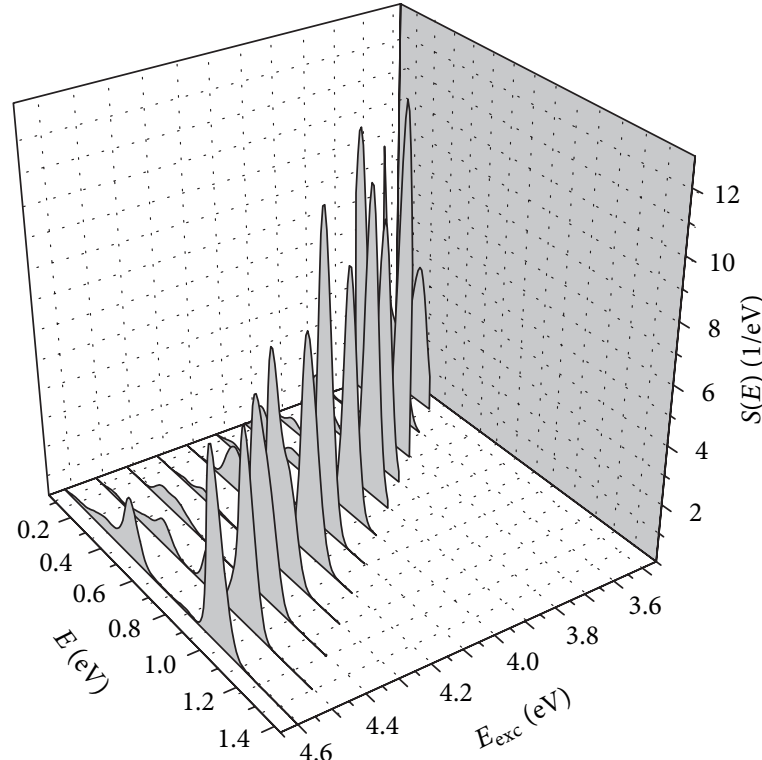

(a)

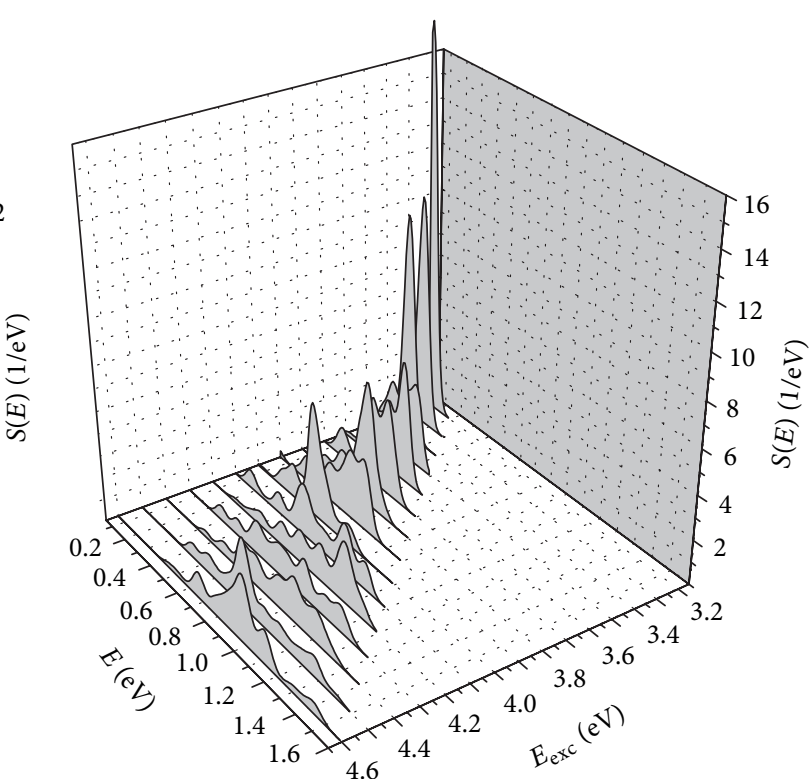

(b)

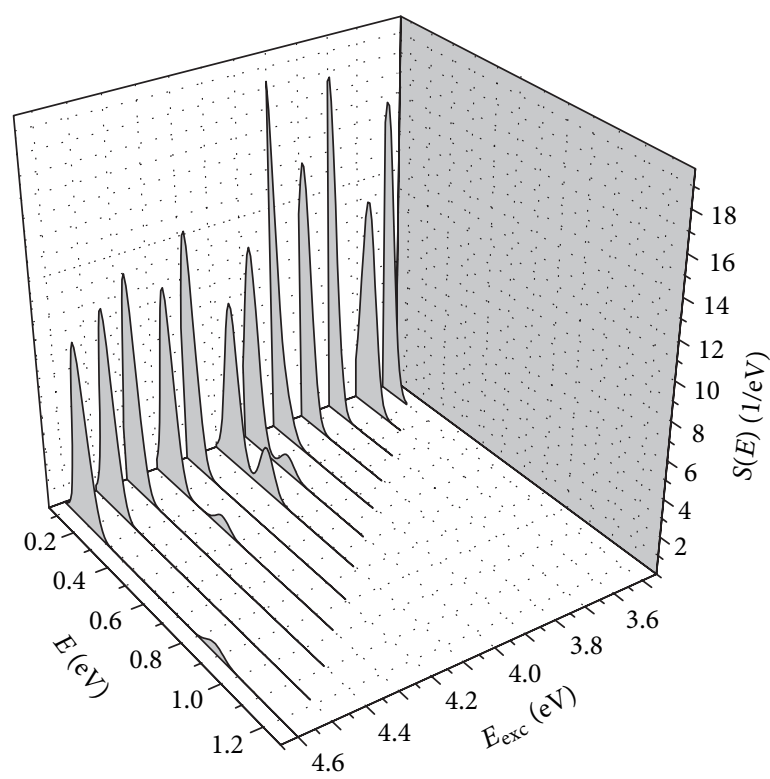

(c)

FIGURE 8: Instantaneous distribution function for anatase (a), rutile (b), and zinc oxide (c) depending on the excess energy of hole $E$, as calculated for the photon energy $E_{\text {exc }}$ from $3.4 \mathrm{eV}$ to $4.6 \mathrm{eV}$.

the constant of electron-phonon coupling, the rate of energy loss, the mean energy of the emitted phonon, and the time of energy loss of a hole. In the case of rutile we find that the time of energy loss of a hole is about $30 \%$ higher than that of an electron. For anatase the time of energy loss of a hole is higher than that of an electron only when excess energy exceeds $0.4 \mathrm{eV}$. Since these data do not directly correlate with experimental results on the rate of oxidative reactions, we conclude that the time of energy loss of a hole is not a major factor in the emergence of the spectral dependence of photocatalytic activity.
In order to elucidate the origin of the spectral dependence of oxidative reactions, we calculated the instantaneous and quasistationary distribution functions of the holes. These functions differ significantly from the analogous functions of excited electrons. The electron distribution functions demonstrate the concentration of excited electrons near zero excess energy that is in the states near the bottom of the conduction band. The distribution functions of holes in anatase reveal the presence of holes in the states more than $\sim 0.8 \mathrm{eV}$ below the top of the valence band. The distribution of holes in rutile shows a significant increase at excess energy above $0.4 \mathrm{eV}$, 
thus reflecting a phenomenon favorable for photocatalytic activity, as this energy increases the oxidative potential of $\mathrm{TiO}_{2}$. The presence of holes in the states below the top of the valence band is consistent with the available experimental data which indicate an increase in the photocatalytic activity at excess energy above $0.2 \mathrm{eV}$. We thus conclude that the major factor responsible for the spectral dependence of photocatalytic activity in $\mathrm{TiO}_{2}$ is a high probability of excitations from the states below the top of the valence band.

We obtained different results for $\mathrm{ZnO}$ which make us conclude that the probability of finding spectral dependence of the photocatalytic activity is less for $\mathrm{ZnO}$ than for $\mathrm{TiO}_{2}$. The major argument in favor of this conclusion is that the calculated distribution function of holes in $\mathrm{ZnO}$ does not extend above the excess energy of $0.2 \mathrm{eV}$.

\section{Conflict of Interests}

The authors declare that there is no conflict of interests regarding the publication of this paper.

\section{Acknowledgments}

The authors acknowledge financial support from the Spanish MICINN (Grant no. FIS2010-19609-C02-01), the Departamento de Educacíon del Gobierno Vasco, the University of the Basque Country (Grant no. GIC07-IT-366-07), and the Presidium of the Ural Branch of Russian Academy of Sciences (Grant no. 12-U-3-1001). The help of Professor L. Baker in the preparation of the paper is also greatly acknowledged. The calculations were performed using the URAN cluster of the Institute of Mathematics and Mechanics of the Russian Academy of Sciences, Yekaterinburg.

\section{References}

[1] M. A. Grela, M. A. Brusa, and A. J. Colussi, "Harnessing excess photon energy in photoinduced surface electron transfer between salicylate and illuminated titanium dioxide nanoparticles," The Journal of Physical Chemistry B, vol. 101, no. 51, pp. 10986-10989, 1997.

[2] M. A. Grela and A. J. Colussi, "Photon energy and photon intermittence effects on the quantum efficiency of photoinduced oxidations in crystalline and metastable $\mathrm{TiO}_{2}$ colloidal nanoparticles," The Journal of Physical Chemistry B, vol. 103, no. 14, pp. 2614-2619, 1999.

[3] M. A. Grela, M. A. Brusa, and A. J. Colussi, "Efficiency of hot carrier trapping by outer-sphere redox probes at quantum dot interfaces," The Journal of Physical Chemistry B, vol. 103, no. 31, pp. 6400-6402, 1999.

[4] T. Morishita, A. Hibara, T. Sawada, and I. Tsuyumoto, "Ultrafast charge transfer at $\mathrm{TiO}_{2} / \mathrm{SCN}^{-}(\mathrm{aq})$ interfaces investigated by femtosecond transient reflecting grating method," The Journal of Physical Chemistry B, vol. 103, no. 29, pp. 5984-5987, 1999.

[5] A. Emeline, A. Salinaro, and N. Serpone, "Spectral dependence and wavelength selectivity in heterogeneous photocatalysis. I. Experimental evidence from the photocatalyzed transformation of phenols," The Journal of Physical Chemistry B, vol. 104, no. 47, pp. 11202-11210, 2000.
[6] Y. Tamaki, A. Furube, R. Katoh et al., “Trapping dynamics of electrons and holes in a nanocrystalline $\mathrm{TiO}_{2}$ film revealed by femtosecond visible/near-infrared transient absorption spectroscopy," Comptes Rendus Chimie, vol. 9, no. 2, pp. 268-274, 2006.

[7] Y. Tamaki, A. Furube, M. Murai, K. Hara, R. Katoh, and M. Tachiya, "Dynamics of efficient electron-hole separation in $\mathrm{TiO}_{2}$ nanoparticles revealed by femtosecond transient absorption spectroscopy under the weak-excitation condition," Physical Chemistry Chemical Physics, vol. 9, no. 12, pp. 1453-1460, 2007.

[8] Y. Tamaki, A. Furube, M. Murai, K. Hara, R. Katoh, and M. Tachiya, "Direct observation of reactive trapped holes in $\mathrm{TiO}_{2}$ undergoing photocatalytic oxidation of adsorbed alcohols: evaluation of the reaction rates and yields," Journal of the American Chemical Society, vol. 128, no. 2, pp. 416-417, 2006.

[9] Q. Shen, K. Katayama, M. Yamaguchi, T. Sawada, and T. Toyoda, "Study of ultrafast carrier dynamics of nanostructured $\mathrm{TiO}_{2}$ films with and without CdSe quantum dot deposition using lens-free heterodyne detection transient grating technique," Thin Solid Films, vol. 486, no. 1-2, pp. 15-19, 2005.

[10] Q. Shen, K. Katayama, T. Sawada, M. Yamaguchi, Y. Kumagai, and $\mathrm{T}$. Toyoda, "Photoexcited hole dynamics in $\mathrm{TiO}_{2}$ nanocrystalline films characterized using a lens-free heterodyne detection transient grating technique," Chemical Physics Letters, vol. 419, no. 4-6, pp. 464-468, 2006.

[11] M. A. Henderson, "A surface science perspective on $\mathrm{TiO}_{2}$ photocatalysis," Surface Science Reports, vol. 66, no. 6-7, pp. 185297, 2011.

[12] V. Zhukov, E. Chulkov, and J. Phys, "Ab initio approach to the excited electron dynamics in rutile and anatase $\mathrm{TiO}_{2}$," Journal of Physics: Condensed Matter, vol. 22, no. 43, Article ID 435802, 2010.

[13] V. P. Zhukov, P. M. Echenique, and E. V. Chulkov, "Two types of excited electron dynamics in zinc oxide," Physical Review BCondensed Matter and Materials Physics, vol. 82, no. 9, Article ID 094302, 2010.

[14] R. Marcus, "On the theory of electron-transfer reactions. VI. Unified treatment for homogeneous and electrode reactions," The Journal of Chemical Physics, vol. 43, pp. 679-701, 1965.

[15] R. A. Marcus, "Reorganization free energy for electron transfers at liquid-liquid and dielectric semiconductor-liquid interfaces," The Journal of Physical Chemistry, vol. 94, no. 3, pp. 1050-1055, 1990.

[16] R. A. Marcus, "Electron transfer reactions in chemistry: theory and experiment (Nobel Lecture)," Angewandte Chemie International Edition in English, vol. 32, no. 8, pp. 1111-1121, 1993.

[17] J. M. Pitarke, V. P. Zhukov, R. Keyling, E. V. Chulkov, and P. M. Echenique, "Ultrafast electron dynamics in metals," ChemPhysChem, vol. 5, no. 9, pp. 1284-1300, 2004.

[18] V. P. Zhukov, E. V. Chulkov, and P. M. Echenique, "Lifetimes of excited electrons in Fe and Ni: first-principles GW and the Tmatrix theory," Physical Review Letters, vol. 93, no. 9, Article ID 096401, 4 pages, 2004.

[19] E. V. Chulkov, A. G. Borisov, J. Gauyacq et al., "Electronic excitations in metals and at metal surfaces," Chemical Reviews, vol. 106, no. 10, pp. 4160-4206, 2006.

[20] S. Baroni, S. de Gironcoli, A. dal Corso, and P. Giannozzi, "Phonons and related crystal properties from densityfunctional perturbation theory," Reviews of Modern Physics, vol. 73, no. 2, pp. 515-562, 2001. 
[21] V. Zhukov, V. Tyuterev, and E. Chulkov, "Electron-phonon relaxation and excited electron distribution in zinc oxide and anatase," Journal of Physics: Condensed Matter, vol. 24, no. 40, Article ID 405802, 2012.

[22] G. M. Eliashberg, "The low temperature specific heat of metals," Soviet Physics, The Journal of Experimental and Theoretical Physics, vol. 16, no. 3, pp. 780-781, 1963.

[23] G. Grimvall, The Electron-Phonon Interactions in Metals, NorthHolland Publisher, Amsterdam, The Netherlands, 1981.

[24] O. Andersen, O. Jepsen, and M. Sob, "Linearized band structure methods," in Electronic Band Structure and Its Applications, M. Yussouff, Ed., vol. 283 of Lecture Notes in Physics, Springer, 1987.

[25] R. Loudon, The Quantum Theory of Light, Oxford University Press, New York, NY, USA, 1983.

[26] V. P. Zhukov and E. V. Chulkov, "Ab initio approach to the rate of radiative electron trapping and electron-hole recombination in B-, C-, and N-doped anatase," Physica Status Solidi B: Basic Research, vol. 249, no. 5, pp. 1063-1071, 2012.

[27] 2010, http://www.quantum-espresso.org and http://www.pwscf .org.

[28] R. Sikora, " $A b$ initio study of phonons in the rutile structure of $\mathrm{TiO}_{2}$," Journal of Physics and Chemistry of Solids, vol. 66, no. 6, pp. 1069-1073, 2005.

[29] O. Jepsen, G. Krier, A. Burkhardt, and O. K. Andersen, The TB-LMTO-ASA program, Max-Planck-Institut für Festkörperforschung, Heisen-bergstr, Federal Republic of Germany, Stuttgart, Germany, 1994.

[30] V. I. Anisimov, J. Zaanen, and O. K. Andersen, "Band theory and Mott insulators: Hubbard U instead of Stoner I," Physical Review B, vol. 44, no. 3, pp. 943-954, 1991.

[31] F. Aryasetiawan and O. Gunnarsson, "Product-basis method for calculating dielectric matrices," Physical Review B, vol. 49, no. 23, pp. 16214-16222, 1994 

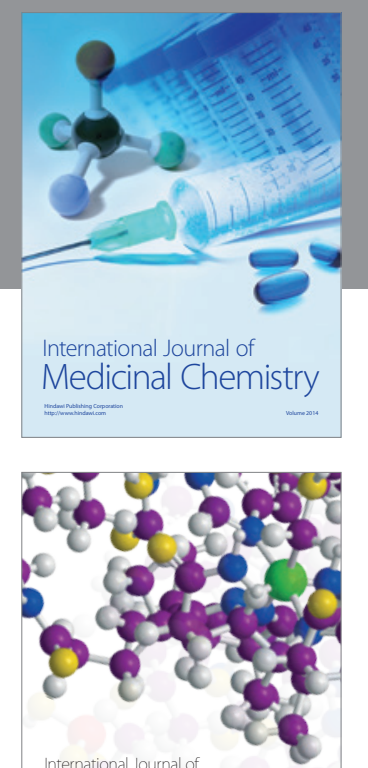

\section{Carbohydrate} Chemistry

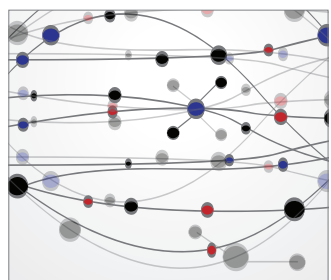

The Scientific World Journal
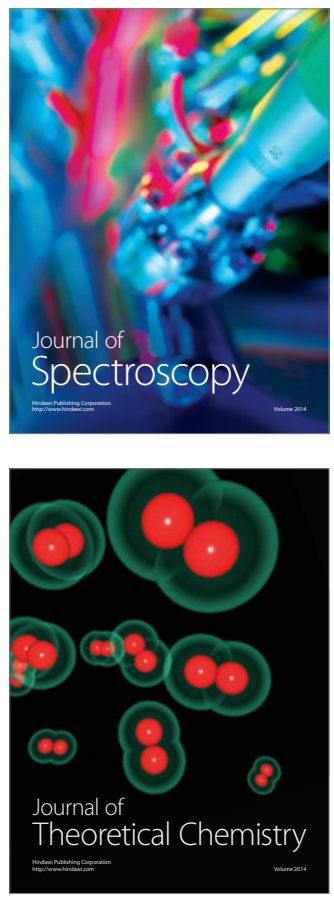
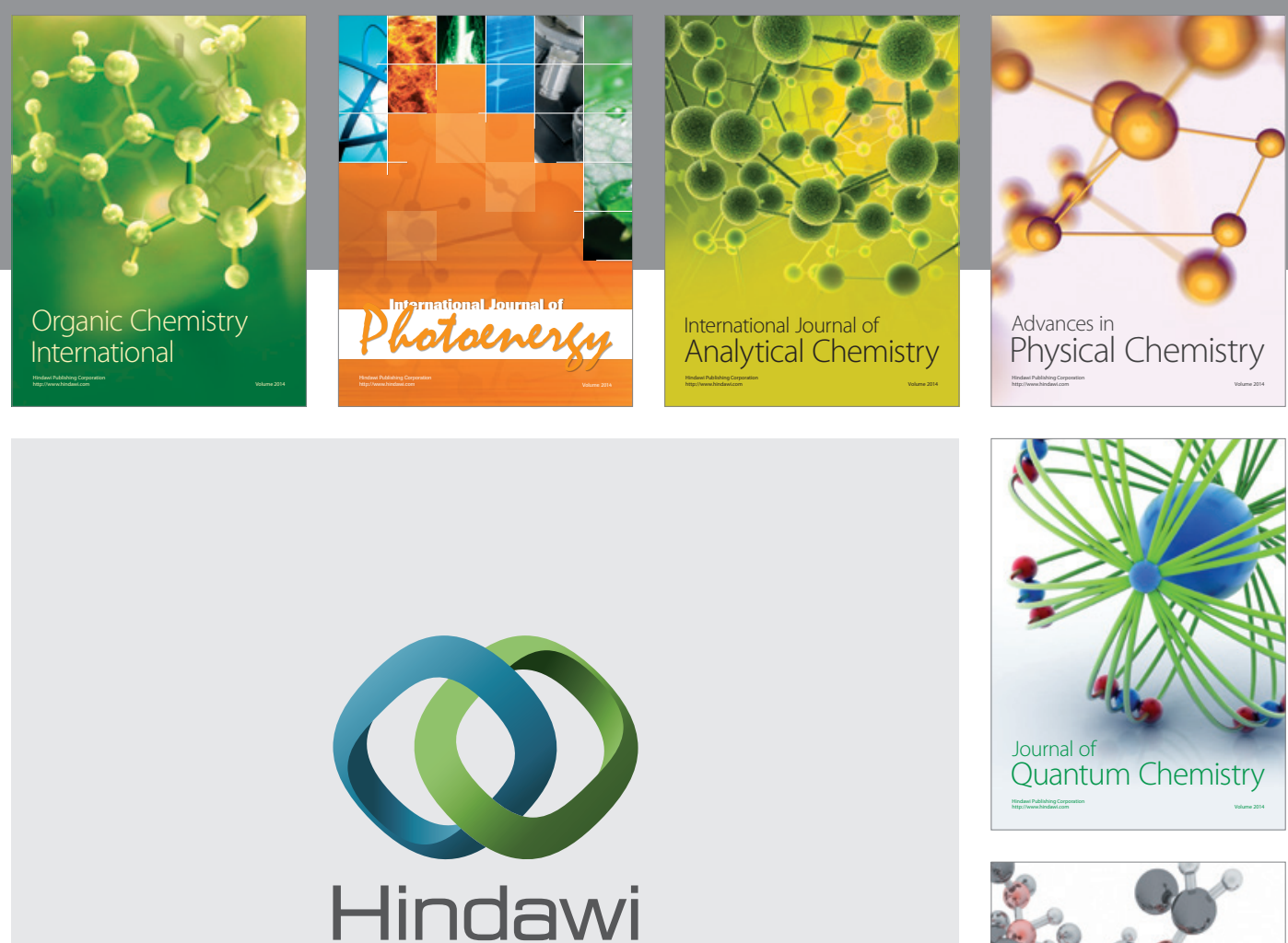

Submit your manuscripts at

http://www.hindawi.com

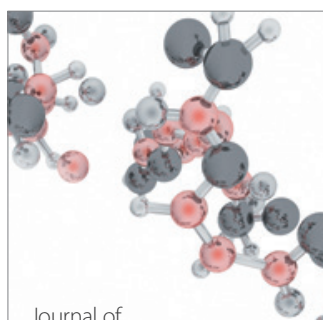

Analytical Methods

in Chemistry

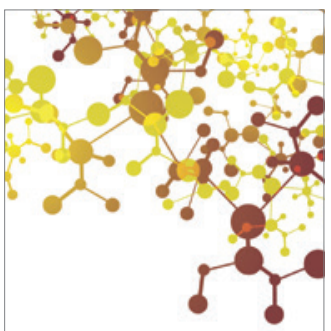

Journal of

Applied Chemistry

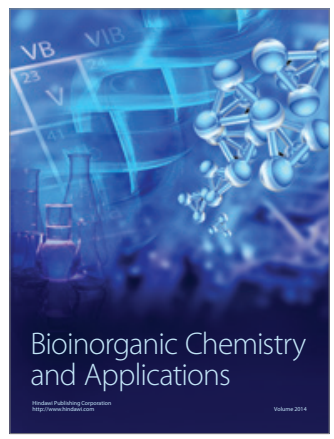

Inorganic Chemistry
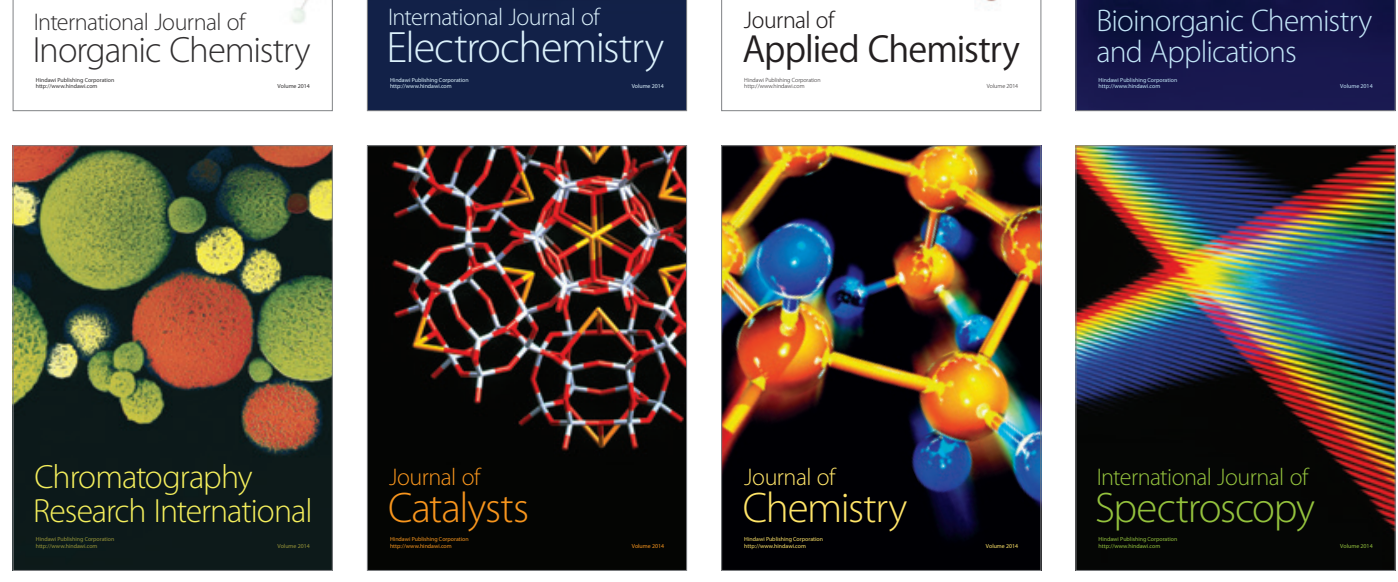